3 


\section{Klaus-Dieter Kossow}

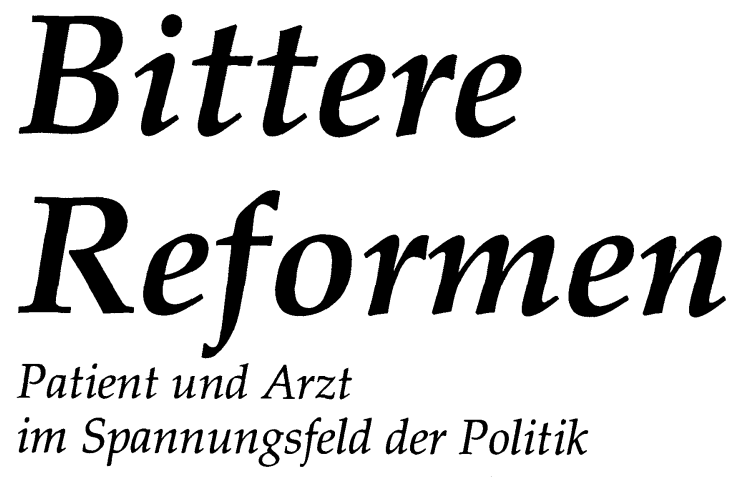

Springer Basel AG 
Herausgegeben von T. Graf-Baumann

CIP-Titelaufnahme der Deutschen Bibliothek

Kossow, Klaus-Dieter:

Bittere Reformen : Patient und Arzt im Spannungsfeld der Politik / Klaus-Dieter Kossow. [Hrsg. von T. Graf-Baumann]. - Basel ; Boston ; Berlin : Birkhäuser, 1990

ISBN 978-3-7643-2391-2 ISBN 978-3-0348-5216-6 (eBook)

DOI 10.1007/978-3-0348-5216-6

Das Werk ist urheberrechtlich geschützt. Die dadurch begründeten Rechte, insbesondere die der Übersetzung, des Nachdruckes, der Entnahme von Abbildungen, der Funksendung, der Wiedergabe auf photomechanischem oder ähnlichem Wege und der Speicherung in Datenverarbeitungsanlagen bleiben, auch bei nur auszugsweiser Verwertung, vorbehalten. Die Vergütungsansprüche gemäß $\S 54$, Abs. 2 UrhG werden durch die "Verwertungsgesellschaft Wort", München, wahrgenommen.

(C) 1990 Springer Basel AG

Ursprünglich erschienen bei Birkhäuser Verlag Basel 1990.

Umschlaggestaltung: Zembsch' Werkstatt, München 


\section{Inhalt}

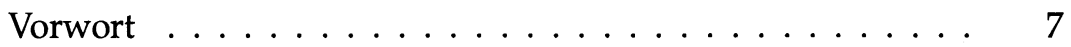

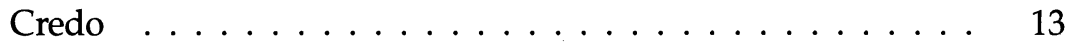

Der Auftrag des Patienten $\ldots \ldots \ldots \ldots$

Die Patient-Arzt-Beziehung . . . . . . . . . . . . . . . . 27

Die Ethik der Patient-Arzt-Beziehung . . . . . . . . . . 48

Medizinische Wissenschaft . . . . . . . . . . . . . . 54

Patient und Arzt in Markt- und Planwirtschaft . . . . . . . . 70

Der Anspruch der Ökonomie an Patient und Arzt . . . . . . . . . . . 74

Praxismarketing ..................... 96

Patient und Arzt von Paragraphen umstellt . . . . . . . . . . 103

Die Patient-Arzt-Beziehung in der kassenärztlichenVersorgung 109

Risiken der Negativ- und Positivlisten . . . . . . . . . . 132

Die kassenärztliche Wirtschaftlichkeitskontrolle . . . . . . . . . 135

Im Namen des Volkes . . . . . . . . . . . . . . . . . . . . 160

Die Ärzteschwemme . . . . . . . . . . . . . . . . . . . . 183

Die Auswirkungen der Ärzteschwemme . . . . . . . . . . . . 191

Patient und Arzt - Wunsch, Wirklichkeit, Ethik und Ökonomie 196

Patient und Arzt - Marionetten der Staatsgewalt . . . . . . . 203

Handlungsaufträge . . . . . . . . . . . . . . . 228

Pragmatik- Ein Problemlösungsansatz für den Arzt . . . . . . . 238

Politische Medizin oder Politik für Patient und Arzt . . . . . 247

Literatur und Anmerkungen . . . . . . . . . . . . . . . 273

Anhang: Tabellen und Abbildungen . . . . . . . . . . 278 
Für Ingrid 


\section{Vorwort}

Dieses Buch habe ich im Jahre 1989 geschrieben. Als ich es im Januar begann, stand ich unter dem Eindruck der Auseinandersetzungen um das Gesundheitsreformgesetz, das am 01.01.1989 in Kraft trat und eine Mauer errichtete zwischen einer Gruppe von Sozialpolitikern der Regierungskoalition und den Bürgern, Ärzten, Apothekern und Arzneimittelherstellern; eine Mauer zwischen der Staatsgewalt und den Betroffenen, denen eine staatliche Ordnung aufgenötigt wurde, deren Bezug zu den Interessen des Gemeinwesens sie nicht zu erkennen vermochten.

Einwände gegen das Gesundheitsreformgesetz während der Diskussion im Gesetzgebungsverfahren wurden durch Regierungsvertreter als Lobbyismus gebrandmarkt. Nun ist das Gesundheitsreformgesetz als 5. Sozialgesetzbuch gegen den Willen der Mehrheit der Betroffenen geltendes Recht. Da aber die Gesundheitsreform weitergehen soll, muß m. E. auch die Diskussion über das Gesundheitswesen fortgesetzt werden. Das Motto "Bonna locuta causa finita" (wenn Bonn gesprochen hat, ist die Angelegenheit erledigt, in Anlehnung an ein geflügeltes Wort im klassischen Rom) paßt vielleicht in die Zeit der römischen Cäsaren, nicht aber in die Demokratie des 20. Jahrhunderts.

Ich meine, daß man gerade jetzt über manches sprechen muß, das im Gesetzgebungsverfahren nicht zur Sprache kommen konnte. Denn dieses Gesetz zählt zu jenen nur schwer erkennbaren Mauern, die auch in der Demokratie die Bürger von den Herrschern trennen können.

Als ich in diesem Buch im Januar 1990 die letzten Korrekturen vornahm, war die Berliner Mauer gefallen. Sie konnte überwunden werden, weil die Macht des Wortes und die fehlende Problemlösungskraft militärischer Operationen einmal mehr Bestätigung fanden. Vom verlorenen Vietnam- und Afghanistan-Krieg, über die Perestroika Gorbatschows und die Oktober-Demonstrationen der Friedensbewegung in der DDR bis zur Wiederherstellung der Einheit von Volkswillen und Handeln der Regierungen Osteuropas zieht sich ein roter Faden.

Diese bewegenden Erlebnisse in der großen Politik des Jahres 1989 haben mich oft zu der Frage geführt, ob mein Thema überhaupt noch 
das Interesse von Lesern finden könnte. Denn diese müßten sich für Fragen der Gesundheitspolitik so sehr interessieren, daß sie derentwegen zu Zeitopfern und Gedankenanstrengungen bereit wären - und dies während die Geschichte sich anschickt, die Verhältnisse in Europa neu zu ordnen.

Ich habe das Manuskript schließlich trotz zeitlicher Enge und folglich fehlender Muße fertiggestellt, weil mich das Thema in meinem Arbeitsalltag nicht mehr losließ.

Beinah täglich bin ich im Sprechzimmer als Hausarzt und Psychotherapeut, aber auch als Mitglied in den Vorständen ärztlicher Körperschaften und Verbänden mit der Frage befaßt, ob die gesetzliche Ordnung der Bundesrepublik den Bedürfnissen und dem Willen der Mehrheit der Bürger entspricht. Dies ist nach meiner Überzeugung im Querschnitt aller Bereiche des Lebens diesèr Gesellschaft sehr wohl der Fall. So gesehen ist die Bundesrepublik Deutschland ein guter Staat, dessen Parlamente und Regierungen in der Zusammenarbeit mit dem Volk gute Arbeit geleistet haben. Deshalb wollen wohl auch so viele Menschen zu uns, die woanders schlechter leben.

Es gibt aber in diesem Lande neben einer exzellenten Wirtschafts-, Finanz-, Sozial- und Rechtspolitik nach meiner Auffassung eine stark verbesserungsbedürftige Gesundheitspolitik. Dies gilt auch für die Kommunikation zwischen den Betroffenen. Ich habe den Eindruck, daß die Verantwortlichen in den Unternehmen der freien Wirtschaft und die Politiker nicht aneinandervorbeireden, wenn sie in Fragen der Wirtschafts- und Finanzpolitik diskutieren. Dies heißt natürlich nicht, daß sie immer das gleiche Interesse verfolgen - im Gegenteil! Es wird aber eine Kommunikationskultur gepflegt, die vom Prinzip der Akzeptanz sachlicher Fakten und der Offenheit bei der Auseinandersetzung über gegensätzliche Interessen geprägt ist. Anders sieht es in der Gesundheitspolitik aus. Hier sind die Ziele verschwommen. Manche Diskutanten vermitteln den Eindruck, daß sie keine klaren Ziele in bezug auf die Pflege der Gesundheit haben.

Was ist Gesundheit; was hält man dafür? Wer ist für das körperliche, seelische und soziale Wohlbefinden verantwortlich? Was ist soziale Befindlichkeit? Äußert sich in ihr eine Seele der Gesellschaft? Was wollen die Kranken und was die Gesunden? Welchen Paradigmen folgen die Gesundheitsberufe, insbesondere die Ärzte und welchen die Politiker?

Mir scheint, daß es zwischen den Ärzten Kommunikationsprobleme gibt, die auf unterschiedlichen Auffassungen über die Ausübung des Arztberufes beruhen. Dies wäre für sich genommen kein Problem. Aber leider hat sich die babylonische Sprachverwirrung von Ärzten 
auf das gesamte Gesundheitswesen und auf die Gesundheitspolitik übertragen.

Es gibt eineobjektiv-naturwissenschaftliche Medizin, die nach technischen Prinzipien Defekte repariert. Aber ist ein derart reparierter Patient gesund? Und ist andererseits jeder objektiv meßbare Defekt in Struktur und Funktionsablauf eines Organismus immer auch Krankheit? Es gibt eine subjektiv am Befinden des einzelnen orientierte Medizin, die es dem Individuum zuschreibt, durch gesunde Lebensführung Gesundheit anzustreben. Wo aber sind die Grenzen der Gesundheitspflege durch das Wort als fraglos wichtigstem didaktischen Werkzeug?

Für jede Meinung gibt es eine Expertenbegründung im Gewande der Wissenschaftlichkeit. Im Konsens treten medizinische Experten nur selten der Öffentlichkeit gegenüber. Deshalb ist Gesundheitspolitik mehr als andere politische Bereiche durch Auseinandersetzungen über Legitimitätsfragen gekennzeichnet. Dem jeweils anders Denkenden, dem Verfechter alternativer Wertungen wird oft unterstellt, er wolle mit seinem Beitrag nicht die Gesundheitspflege und das Wohl des Kranken, sondern seine eigenen Interessen fördern.

Von Ehrenberg bis Blüm haben alle Sozialminister Klage über die Lobby in Weiß geführt. Es soll nicht bestritten werden, daß es ärztliche Eigeninteressen in der Gesundheitspolitik ebenso gibt, wie bei kranken und gesunden Bürgern, Apothekern, Krankenhausträgern und Arzneimittelherstellern. Alle am Gesund heitswesen Beteiligten haben Eigeninteressen. Warum sollten gerade die Politiker davon ausgenommen sein? Es besteht immerhin die Möglichkeit, daß sich auch Politiker nicht nur dem Gemeinwohl verpflichtet fühlen, wenn sie ihre Wiederwahl anstreben. Nicht alle gesundheitspolitischen Handlungen lassen einen Bezug zum Interesse der Bürger erkennen.

Was ist der Gesundheitspflege beim einzelnen und in der Gesamtgesellschaft dienlich? Was ist notwendig, um dem Bürger zu helfen, wenn er sich bei Krankheitsproblemen nicht selbst helfen kann? Die Antwort auf diese Frage wird in der Gesundheitspolitik meist von denen gegeben, die selbst nie einen Patienten verantwortlich behandelt haben. Hier liegt ein wichtiger Unterschied zwischen der Wirtschafts- und Finanzpolitik, die von Ökonomen wesentlich gestaltet wird. Auch in der Rechtspolitik dominieren Juristen. Demgegenüber sind Ärzte fast ohne Bedeutung für die Gesundheitspolitik - wenn man einmal davon absieht, daß sie den politischen Willen von Nichtärzten durch Auftragsgutachten absichern.

Der vergleichsweise geringe Stellenwert der Ärzte in politischen Dingen hat viele Gründe unter denen mir drei besonders wichtig erscheinen: 
1) Ärzte sind sich derzeit untereinander nicht darüber einig, wie Medizin organisiert werden sollte. Sie befinden sich in einem sog. Paradigmenstreit, zu dem dieses Buch einen Kommentar liefert.

2) Arzte hielten in den letzten Jahrzehnten die Behandlung von Patienten und die Beschäftigung mit Kunst, Kultur und Freizeitgestaltung für sinnvoller als die Politik. Der Leser dieses Buches könnte einen Beitrag dazu leisten, daß sich dies ändert.

3) Ärzte haben sich als Lobby in Weiß brandmarken und das Gesetz politischen Handelns abkaufen lassen. Dieses Buch wird zeigen, daß bei gesundheitspolitischen Auseinandersetzungen Beteiligte selten frei sind von Eigeninteressen, und daß dies kein Argument gegen die Beteiligung der Ärzte an der gesundheitspolitischen Diskussion ist. Bürger zahlen mit verminderten Gesundheitschancen dafür, wenn Ärzte sich einschüchtern und aus der Politik verdrängen lassen.

Patient und Arzt sind von Richtlinien und gesetzlichen Regelungen umstellt. Sie haben gleichermaßen das Recht an deren Inhalt mitzuwirken. Geschieht dies nicht, dann werden jene bürokratischen Normen, nach denen Patient und Arzt sich zu richten haben, zu einer Mauer zwischen Regierung und Parlament einerseits und den Bürgern andererseits. Auf der Seite der Herrschenden entstehen dann Ideologien, die zwar systembestimmend werden, aber keinen realen Bezug mehr zu den Bedürfnissen der Bürger haben. Dieser Prozeß ist meiner Überzeugung nach schon weit fortgeschritten, weil die Sozialgesetzgebung von Ehrenberg bis Blüm gegen wesentliche Interessen von Bürgern, Ärzten, Apothekern, Pharmaherstellern und Krankenhausträgern gerichtet ist, aber mit den Machtinteressen der Politiker durchaus konform geht.

Dies konnte geschehen, weil Regierungsmitarbeiter und Parlamentarier die Lebenswirklichkeit des kranken Bürgers nicht ausreichend zur Kenntnis genommen haben. Die Lebenswirklichkeit aber konnte von Ärzten nicht ausreichend vermittelt werden, weil deren Beteiligung am politischen Leben zu schwach ist. Dieses Buch möchte die gesundheitspolitische Diskussion anregen, damit sich dies ändert und damit der Überbau des Systems gesundheitlicher Sicherung nicht der Basis entrückt. Diese wird durch Bürger und praktische Heilberufe, durch Patient und Arzt gebildet.

In der Begegnung zwischen Patient und Arzt geht es nicht in erster Linie um die Frage der Wissenschaft, was wahr ist und was nicht, sondern um den zweckmäßigen also "pragmatischen" Weg zur Lösung der Probleme der Patienten. Wichtig sind ferner Fragen der Bewertung. Was Patient und Arzt für gut und böse halten, unterliegt historischen Veränderungen, die oft schleichend verlaufen, und die 
die Patient-Arzt-Beziehung mehr beeinflussen, als alles andere. Dies gilt ganz generell für das Leben der Menschen.

Ein Wappenspruch der alten Bremer Kaufmannschaft lautete: "Seefahrt tut not, Leben ist nicht notwendig" (navigare necesse est, vivere non est necesse). Hieraus spricht eine am Gemeinwohl orientierte religiöse Lebensauffassung, die davon ausgeht, daß unser tägliches Tun und Treiben einen höheren Sinn hat als nur den der individuellen Bedürfnisbefriedigung und Selbstverwirklichung.

Aus heutiger Sicht beinhaltet dieser alte bremische Spruch eine Ungeheuerlichkeit: Die Seefahrt wird für wichtiger erachtet als das Leben des einzelnen, weil sie die Existenzgrundlage der bremischen Wirtschaftsgemeinschaft ist.

Heute begegnen wir einem Zeitgeist, der zu umgekehrter Konsequenz führt: Im Interesse individueller Selbstverwirklichung werden von den Bürgern Rechte geltend gemacht und von den Organisatoren der Gesellschaft, den Politikern, garantiert, ohne daß die Erfüllung der Ansprüche inhaltlich und finanziell durch die Gesellschaft auch wirklich gewährleistet werden kann.

Es kommt daher zu einer Rückdelegation der Arbeit für die Ansprüche an jene, denen die Politiker mit Heilsverheißungen und der Gewährung von Rechten gegenüber getreten sind: An die Bürger, die Beiträge und Steuern aufwenden müssen und an Berufstätige, die sich mit einem zunehmenden Dickicht an bürokratischen Auflagen konfrontiert sehen.

Im Gesundheitswesen ist es die Patient-Arzt-Beziehung, die zunehmend solchen Belastungen ausgesetzt ist; in ihr soll alles ausgeglichen werden, was an fehlender Ausgewogenheit zwischen Rechten und Pflichten in der Gesundheitspolitik verankert wurde. Hinzu kommt, daß expandierende Sozialetats zur Staatsverschuldung beitragen, wodurch selbst künftige Generationen an der heutigen Gesundheitspflege beteiligt werden.

Übrigens sind gerade die Bremer Bürger an diesem Verschuldungsprozeß zu Lasten der Steuerzahler künftiger Generationen besonders beteiligt. Ihr Gemeinwesen gehört zu denjenigen mit der höchsten Pro-Kopf-Verschuldung in der Bundesrepublik Deutschland. Die im Wappenspruch öffentlich verkündigte Moral wird in der Alltagspolitik heute nicht mehr zur Kenntnis genommen. Das hinderte aber gerade die Bremer Politiker nicht daran, Patient und Arzt besonders intensiv mit moralischen Postulaten zu überziehen.

Zwischen Politikern und ihren Beratern, insbesondere den Ökonomen und Juristen sowie ihren Willens- und Meinungsvermittlern, den Journalisten einerseits - und manchen Patienten und Ärzten anderer- 
seits - haben sich Verständigungsprobleme entwickelt. Die eine Seite versteht die Sprache der anderen rein begrifflich nicht mehr, aber selbst wo dies nicht der Fall ist, entwickelt sich kein beobachtbares Verständnis der einen Seite für die Aufgaben der anderen.

Insbesondere die Auseinandersetzungen um das Gesundheitsreformgesetz sind hierfür ein konkretes Beispiel. Patienten und Ärzte sowie der Bundesminister für Arbeit und Soziales haben ihre Auffassung zum Gesetzentwurf auf Plakaten ausgetauscht. Statt dessen wäre eine differenzierte Diskussion über Pflichten und Rechte des Bürgers, des Patienten und des Arztes bis zum möglichst weitgehenden Interessenausgleich nötig gewesen. Demgegenüber wurden die meisten Ärzte, auch solche, die sich um eine differenzierte Würdigung der Absichten des Ministers bemüht haben, als "Lobbyisten" beschimpft.

Unmaß und Unangemessenheit im Umgang mit dem ärztlichen Handlungsauftrag sind nunmehr auch auf Regierungsebene zu finden. Bei manchen Staatsanwaltschaften und in der Tendenzpresse sind wir Ärzte dies schon seit längerem gewohnt.

Nach wie vor bleibt aber die Tatsache bestehen, daß Hilfe im Krankheitsfall und selbst Gesundheitspflege ohne Ärzte nicht möglich ist. Nach wie vor gibt es Menschen mit Gesundheitsproblemen, die eine Vertrauensbeziehung zum Arzt suchen, weil sie mit ihrer Lebensangst, ihren Schmerzen und ihren verminderten Lebenschancen selbst nicht mehr fertig werden. Eine Patient-Arzt-Beziehung, die mit politischen, ökonomischen, juristischen und selbst wissenschaftlich-technischen Belastungen fertig werden muß, steht im Bezug auf ihre Problemlösungskraft für die Beschwernisse des Patienten zunehmend in Frage.

Dieses Buch ist für neugierige, frustrierte oder kritische Menschen bestimmt, die nicht mehr darauf vertrauen, daß es für sie noch verläßliche und hilfreiche Ärzte gibt, die aber dennoch diese vermeintliche Situation ändern möchten.

Ferner sind Ärzte und Gesundheitspolitiker angesprochen, damit sie sich auf die Diskussion über die noch vor uns liegenden Reformen des Gesundheitswesens vorbereiten können, wenn sie hierfür Anregungen suchen.

Achim-Baden im Dezember 1989

Klaus-Dieter Kossow 